\title{
Determinação de espécies bioindicadoras de resíduos de herbicidas auxínicos
}

\author{
Daiana Pereira dos Santos ${ }^{1}$, Renan Rodrigues Braga², Fernanda Aparecida Rodrigues Guimarães ${ }^{3}$, \\ Ana Beatriz Rocha de Jesus Passos ${ }^{4}$, Daniel Valadão Silva ${ }^{5}$, José Barbosa dos Santos 6 , Marcela Carlota Nery ${ }^{7}$
}

\begin{abstract}
RESUMO
Herbicidas que apresentam atividade residual no solo podem se tornar problema para os cultivos agrícolas, pela possibilidade de intoxicar culturas sucessoras. No caso dos hormonais, há grande dificuldade na determinação de resíduos pela técnica do bioensaio, em função da variação na resposta em crescimento, apresentada pela espécie indicadora. Neste trabalho, objetivou-se determinar espécies e variáveis sensíveis aos herbicidas 2,4 D e picloram, para indicação de resíduos no solo. Para isso, realizaram-se dois bioensaios, sendo que cada um, constituiu-se de 60 tratamentos, em esquema fatorial, compostos pela combinação de três espécies vegetais (Lycopersicon esculentum, Cucumis sativus e Beta vulgaris), dois substratos (areia e amostra de solo) e dez subdoses do herbicida $(0 ; 3 ; 6 ; 12$; 22,$5 ; 47 ; 94 ; 187,5 ; 375$ e $\left.750 \mathrm{~g} \mathrm{ha}^{-1}\right)$ e $\left(0 ; 3 ; 6 ; 9 ; 18 ; 30 ; 60 ; 123 ; 246 \mathrm{e} 501 \mathrm{~g} \mathrm{ha}^{-1}\right)$ para o 2,4-D e picloram, respectivamente. $\mathrm{O}$ delineamento experimental utilizado para ambos os ensaios foi em blocos casualizados, com quatro repetições. $\mathrm{O}$ aumento das doses dos herbicidas provocou reduções nas variáveis altura de planta, matéria seca e, aumento da intoxicação visual das espécies avaliadas. A intoxicação visual mostrou-se a variável mais adequada para determinação de baixos níveis de resíduos de 2,4 D e picloram no solo. B. vulgaris foi a espécie com maior sensibilidade ao 2,4 D enquanto que L. esculentum foi a espécie mais sensível ao picloram.
\end{abstract}

Palavras-chave: planta teste, Lycopersicon esculentum, Cucumis sativus, Beta vulgaris.

\begin{abstract}
Determination of bioindicators of auxinic herbicides residues

Herbicides that present soil residual activity may become a problem in agricultural crops due to the possibility of crops successors intoxication. It is hard to detect residues of hormonal herbicides using the bioassay technique, due to the variation in the growth response of the indicator plant. The objective of this work was to select plants and variables susceptible to the herbicides 2,4-D and picloram for soil residue indication..To make it possible, two bioassays were performed,, where each of them consisted of 60 treatments in a factorial scheme with the combination of three plant species (Lycopersicon esculentum, Cucumis sativus and Beta vulgaris), two substrates (sand and soil sample) and 10 subdoses of herbicide $\left(0,3,6,12,22.5,47,94,187.5,375\right.$ and $\left.750 \mathrm{~g} \mathrm{ha}^{-1}\right)$ and $(0,3,6,9,18,30,60,123 ; 246$ and 501
\end{abstract}

Recebido para publicação em 29/12/2011 e aprovado em 07/03/2013.

${ }^{1}$ Engenheira-Agrônoma. Departamento de Agronomia, Universidade Federal dos Vales do Jequitinhonha e Mucuri, Rodovia MGT 367, Km 583, 5000, Alto da Jacuba, 39100-000, Diamantina, Minas Gerais, Brasil. daianaagro@ hotmail.com

${ }^{2}$ Engenheiro-Agrônomo. Departamento de Agronomia, Universidade Federal dos Vales do Jequitinhonha e Mucuri, Rodovia MGT 367, Km 583, 5000, Alto da Jacuba, 39100-000, Diamantina, Minas Gerais, Brasil. granderenan@gmail.com

${ }^{3}$ Engenheira-Agrônoma. Departamento de Fitotecnia, Universidade Federal de Viçosa, Campus Viçosa, Avenida Peter Henry Rolfs, s/n, 36570-000, Viçosa, Minas Gerais, Brasil. fernanda.guimaraes@ufv.br

${ }^{4}$ Química, Mestre. Departamento de Química, Universidade Federal de Viçosa, Campus Viçosa, Avenida Peter Henry Rolfs, s/n, 36570-000, Viçosa, Minas Gerais, Brasil. anabiapassos@yahoo.com.br (autor para correspondência).

${ }^{5}$ Engenheiro-Agrônomo, Mestre. Departamento de Fitotecnia, Universidade Federal de Viçosa, Campus viçosa, Avenida Peter Henry Rolfs, s/n, 36570-000, Viçosa, Minas Gerais, Brasil.danielvaladaos@yahoo.com.br

${ }^{6}$ Engenheiro-Agrônomo, Doutor. Departamento de Agronomia, Universidade Federal dos Vales do Jequitinhonha e Mucuri, Rodovia MGT 367, Km 583, 5000, Alto da Jacuba, 39100-000, Diamantina, Minas Gerais, Brasil. jbarbosasantos@yahoo.com.br

${ }^{7}$ Engenheira-Agrônoma, Doutora. Departamento de Agronomia, Universidade Federal dos Vales do Jequitinhonha e Mucuri, Rodovia MGT 367, Km 583, 5000, Alto da Jacuba, 39100-000, Diamantina, Minas Gerais, Brasil. nery.marcela@gmail.com

Rev. Ceres, Viçosa, v. 60, n.3, p. 354-362, mai/jun, 2013 
$\mathrm{g} \mathrm{ha}^{-1}$ ) for 2,4-D and picloram, respectively. The experimental design for both trials was randomized blocks with four replications. Increasing doses of the herbicide caused reductions in plant height, total dry matter and visual intoxication of the assessed species. The visual intoxication was the most suitable variable for the determination of low residues levels of D 2.4 and picloram in soil. B. vulgaris was the most sensitive species to 2,4 D and L. esculentum was the most sensitive species to picloram,

Key words: test plant, Lycopersicon esculentum, Cucumis sativus, Beta vulgaris.

\section{INTRODUÇÃO}

O controle químico de plantas daninhas constitui prática indispensável para a agricultura em larga escala, por sua pouca dependência de mão de obra e rapidez de aplicação. Os herbicidas, depois de aplicados, podem ser absorvidos pela parte aérea da planta, ou atingirem o solo. Quando atingem o solo, inicia-se o processo de sua redistribuição e degradação, o qual pode ser extremamente curto, como o que ocorre para algumas moléculas simples e não persistentes, ou perdurar por meses ou anos, para compostos altamente persistentes (Filizola et al., 2002).

De acordo com Procópio et al. (2008), herbicidas que apresentam atividade residual no solo são importantes insumos para a garantia da produtividade das culturas comerciais, principalmente para aquelas que apresentam extenso período total de prevenção da interferência das plantas daninhas. Contudo, após o término desse período, que muitas vezes coincide com o fechamento do dossel do cultivo agrícola, a presença do herbicida no solo pode- se tornar indesejável, podendo resultar em carryover (Belo et al., 2007).

Os herbicidas auxínicos ou mimetizadores de auxina foram introduzidos na agricultura no final da década de 1940, sendo os primeiros herbicidas orgânicos seletivos para o controle de plantas daninhas. Ainda hoje são extensivamente utilizados em culturas de arroz, milho, trigo e cana-de-açúcar e em pastagens (Thill, 2003).

Dentre os herbicidas deste grupo, o 2,4-D (ácido 2,4Diclorofenoxiacético) e o picloram (ácido 4-amino 3,5,6 tricloro-2-piridinacarboxílico) possuem o maior número de produtos registrados para aplicação na agricultura (Brasil, 2013). Esses produtos são latifolicidas, sendo que o 2,4-D apresenta persistência, de curta a média, nos solos, podendo, segundo Silva et al. (2007a), causar intoxicação em espécies sensíveis, como soja, feijão, algodão e outras dicotiledôneas, quando estas são cultivadas em áreas nas quais o herbicida foi aplicado. O picloram apresenta como principal problema a possibilidade de contaminação de organismos não alvo, como as culturas estabelecidas em sucessão, e a contaminação ambiental ocasio- nada por sua lixiviação para camadas mais profundas no perfil do solo, podendo atingir cursos de águas (Santos et al., 2007).

Uma das técnicas mais comuns para identificar e quantificar um herbicida no solo ou na água é por meio de bioensaios, utilizando-se plantas indicadoras que apresentam fácil cultivo, rápido desenvolvimento e alta sensibilidade aos herbicidas avaliados (Nyffeler et al., 1982; Souza, 1999). Segundo Thill (2003), sintomas de intoxicação produzidos nas folhas de várias dicotiledôneas, por herbicidas auxínicos, são facilmente caracterizados e, por isso, são muito usados para detectar resíduos desses herbicidas no solo. A técnica do bioensaio tem sido questionada para aplicação em ambientes com resíduo de herbicidas hormonais. Como esses produtos interferem na síntese de proteínas e enzimas, a variação da concentração na solução do solo produz diferentes efeitos no desenvolvimento da bioindicadora, dificultando a correlação entre aumento de doses e de injúria (Santos et al., 2007).

Considerando-se o tempo de permanência desses herbicidas no solo e a possibilidade de contaminação de culturas sucessoras, este trabalho objetivou determinar plantas e variáveis sensíveis aos herbicidas 2,4-D e picloram, para indicação de resíduo no solo.

\section{MATERIAL E MÉTODOS}

O experimento foi conduzido em casa de vegetação com condições controladas de temperatura e umidade. Foram realizados dois bioensaios, sendo cada um constituído de 60 tratamentos, em esquema fatorial, compostos pela combinação de três espécies vegetais, Lycopersicon esculentum (tomate), Cucumis sativus (pepino) e Beta vulgaris (beterraba); dois substratos (areia fina e amostra de solo) e dez subdoses do herbicida $(0 ; 3 ; 6 ; 12 ; 22,5 ; 47$; $94 ; 187,5 ; 375$ e $\left.750 \mathrm{~g} \mathrm{ha}^{-1}\right)$ e $(0 ; 3 ; 6 ; 9 ; 18 ; 30 ; 60 ; 123 ; 246$ e $501 \mathrm{~g} \mathrm{ha}^{-1}$ ), para o 2,4-D e picloram (respectivamente). $\mathrm{O}$ delineamento experimental utilizado para ambos os ensaios foi em blocos casualizados, com quatro repetições.

As espécies vegetais foram cultivadas em areia lavada (substrato inerte) e solo, visando a compará-los para 
estimativa da Relação de Adsorção (RA), utilizando-se a metodologia proposta por Souza (1996), que expressa quantas vezes a concentração necessária para reduzir em $50 \%$ o crescimento da planta-teste $\left(\mathrm{I}_{50}\right)$ é sorvida por determinado substrato, em relação ao obtido na areia lavada, considerada como substrato inerte:

$\mathrm{RA}=\frac{\mathrm{I}_{50} \text { solo }-\mathrm{I}_{50} \text { areia }}{\mathrm{I}_{50} \text { areia }}$

Para essa relação, a regressão adotada foi aquela oriunda dos dados de intoxicação visual, pela maior sensibilidade à indicação de resíduos dos herbicidas no solo. Para a obtenção do substrato inerte, a areia $(0,05 \mathrm{~mm}$ a $2 \mathrm{~mm})$ foi lavada em água corrente, para retirada das impurezas; posteriormente, realizou-se a incubação do material em ácido sulfúrico diluído (10\%), por 24 horas. Em seguida, procedeu-se a nova lavagem do material em água corrente, até completa eliminação do resíduo ácido, seguido pela correção do $\mathrm{pH}$ final para neutralidade $(\mathrm{pH}$ 7), por meio da adição controlada de solução de hidróxido de sódio. Então a areia foi secada em estufa, a $150^{\circ} \mathrm{C}$.

O solo utilizado foi classificado como Latossolo Vermelho-Amarelo distrófico típico, textura argilosa (56\% argila, $6 \%$ silte e $38 \%$ areia). A análise química apresentou $\mathrm{pH}$ (água) de 5,3; teor de matéria orgânica de 1,6 daq kg-1; $\mathrm{P}, \mathrm{K}, \mathrm{Ca}, \mathrm{Mg}, \mathrm{Al}, \mathrm{H}+\mathrm{Al}$ e $\mathrm{CTC}_{\text {efetiva }}$ de 0,$2 ; 10 ; 0,5 ; 0,2 ; 0,3$; 6,5 e $1,2 \mathrm{cmol}_{\mathrm{c}} \mathrm{dm}^{-3}$, respectivamente.

Os vasos, com capacidade para $100 \mathrm{~cm}^{3}$, foram preenchidos com os substratos (solo e areia) e dispostos em fileira para receberem o herbicida, em aplicação por meio de pulverizador costal pressurizado a $\mathrm{CO}_{2}$, calibrado na pressão 40 lpf e pontas de pulverização 8003 , aspergindose o equivalente a $150 \mathrm{~L} \mathrm{ha}^{-1}$ de calda. Após 24 horas da aplicação dos herbicidas, realizou-se a semeadura das espécies. Foram semeadas dez sementes de tomate e cinco sementes de beterraba e de pepino por vaso.

Após a etapa de semeadura, realizaram-se, a cada dia, duas irrigações, com solução balanceada e completa de macro e micronutrientes, com as seguintes concentrações ( $\mathrm{mg} \mathrm{L}^{-1}$ ): macronutrientes: 182 de N-NO $; 42$ de N-NH $; 31$ de $\mathrm{P} ; 195$ de $\mathrm{K} ; 120$ de $\mathrm{Ca} ; 48$ de $\mathrm{Mg} ; 64$ de $\mathrm{S}-\mathrm{SO}_{4}$; e micronutrientes: 0,5 de B; 0,02 de $\mathrm{Cu}$; 5,0 de Fe; 0,5 de Mn; 0,05 de Zn e 0,01 de Mo (Salvador et al., 1999).

Aos 17 dias após semeadura, foram feitas avaliações visuais de intoxicação, com uso de uma escala percentual de notas, variando entre 0 (zero) e 100 (cem), em que 0 implica ausência de quaisquer injúrias e, 100, a morte da planta (ALAM, 1974). Foram avaliadas, também, altura média de plantas e matéria seca total, sendo essa última determinada após secagem em estufa $\left(72 \mathrm{~h}\right.$, a $\left.75^{\circ} \mathrm{C}\right)$. Os dados foram convertidos em percentagem (proporção relativa à testemunha, que recebeu valor de 100\%). Foram estimadas as curvas de dose-resposta para cada compos- to com as plântulas específicas nos dois substratos (solo e areia lavada).

Para interpretação dos dados, empregou-se a análise de variância, utilizando-se do teste $\mathrm{F}(<0,05)$. Foram determinadas regressões, utilizando-se o modelo sigmoidal logístico de quatro parâmetros, por meio do pacote estatístico Sigmaplot, a partir da regressão não linear, como proposto por Seefeldt et al., (1995):

$$
y=y 0+\frac{a}{1+\left(\frac{X}{I_{50}}\right)^{b}}
$$

Nesse modelo, $x$ e $y$ são as variáveis independente e dependente da equação, respectivamente, $a$ representa a diferença entre os limites superior e inferior da curva, $b$ representa a declividade da curva, $I_{50}$ é a dose que proporciona $50 \%$ do valor da variável dependente e y0 é a média da resposta sob doses elevadas (Seefeldt et al., 1995).

\section{RESULTADOS E DISCUSSÃO}

Os valores observados para altura de planta (ALT) das espécies foi inversamente proporcional ao aumento da dose do herbicida 2,4-D, tanto quando aplicado na areia ou no solo (Figura 1), sendo esta redução mais acentuada para tomate (Figura 1b), espécie que apresentou menores coeficientes de declividades das curvas (Tabela 1). No entanto, foram observados diferentes níveis de tolerância das plantas ao produto. A espécie que apresentou maior sensibilidade em relação a esta variável foi a beterraba (Figura 1a), para a qual a utilização das doses 102,58 e 3,55 $\mathrm{g} \mathrm{ha}^{-1}$ de 2,4$\mathrm{D}$, em solo e areia, respectivamente, foram suficientes para reduzir em $50 \%$ a altura das plantas.

A aplicação do 2,4-D reduziu o acúmulo de matéria seca total (MST) das espécies avaliadas (Figura 2). Foram observadas quedas mais acentuadas da MST em beterraba (Figura 2a), quando utilizada a areia como substrato, e em tomate, quando utilizado solo. Para esta variável verifica-se que, para o pepino (Figura 2c), a dose de 23,25g ha $^{-1}$ foi suficiente para inibir em $50 \%$ o acúmulo de massa da matéria seca total, demonstrando ser, dentre as espécies estudadas, aquela com maior sensibilidade a baixas concentrações deste herbicida, quando foi utilizado como substrato solo. Entretanto, em material inerte, tomate foi a espécie mais sensível, sendo que a dose de 14,05 $\mathrm{g} \mathrm{ha}^{-1}$ foi suficiente para a redução de $50 \%$ do acúmulo de matéria seca total.

A sensibilidade do pepino a outros herbicidas já tem sido considerada em bioensaios para estimar níveis de resíduos de trifloxysulfuron (Monquero et al., 2008); contudo, essa mesma espécie não foi efetiva, comparada com Brassica oleraceae, na indicação de resíduos de Stryphnodendron polyphyllum (barbatimão), proposto 
como bio-herbicida para o controle de dicotiledôneas (Petacci et al., 2001). Vários trabalhos usam essa espécie como bioindicadora de resíduos de sulfonilureias, em função da sua sensibilidade (Santos et al., 2007; Silva et al., 2007b). Contudo, a falta de correlação entre variação na produção de massa e de altura de plantas, para pequenas doses de herbicidas hormonais, tem dificultado o uso do pepino para estimar os resíduos de auxinas (Santos et al., 2007).
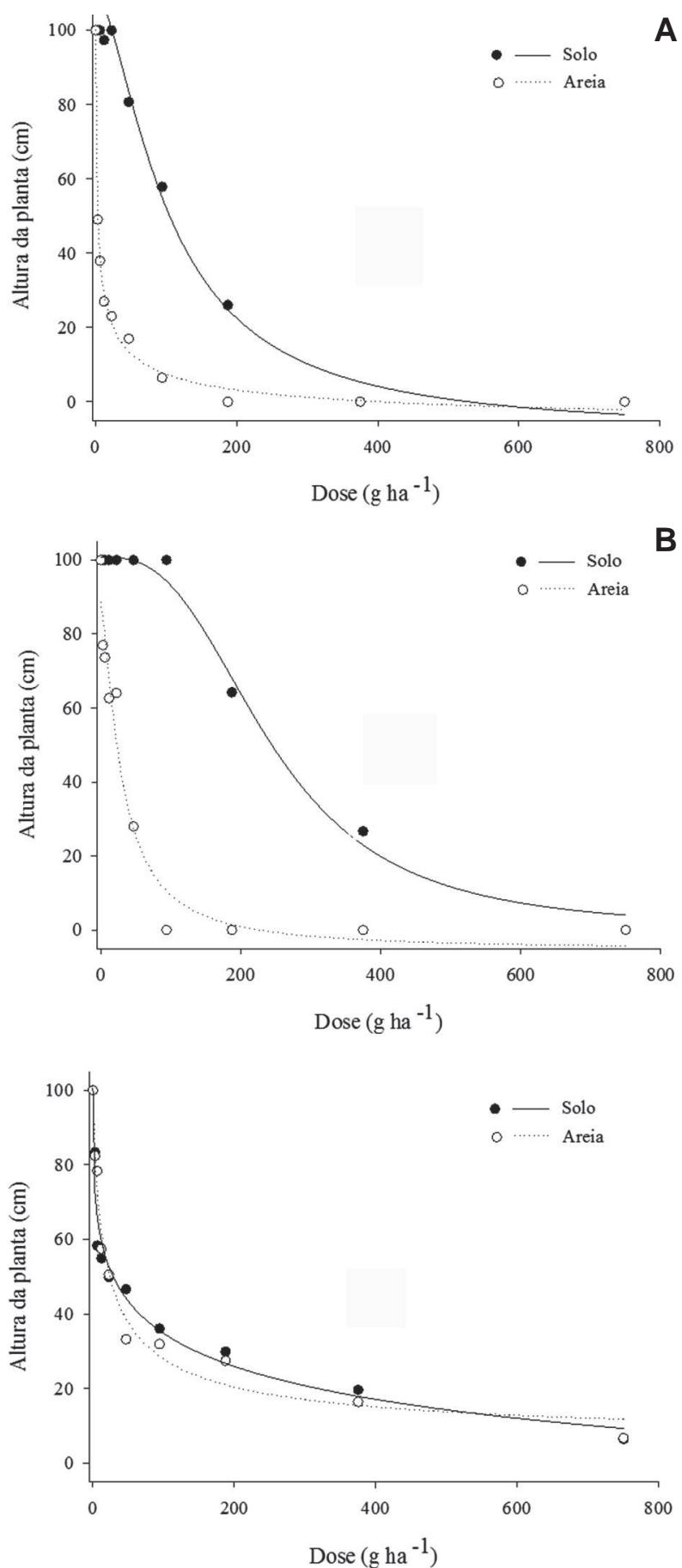

Figura 1. Curva de dose-resposta para altura de plantas de beterraba (a), tomate (b) e pepino (c) em solo e em areia, em função de doses crescentes do herbicida 2,4-D
Em relação à intoxicação visual (INT), as espécies apresentaram comportamento semelhante em respostas à presença do 2,4-D no solo e na areia (Figura 3). Foram observados, tanto para o 2,4-D, quanto para o picloram, o encarquilhamento e o dobramento das bordas das folhas, com posterior clorose e necrose das folhas e caules de todas as espécies bioindicadoras (Figura 4). Os menores valores de $I_{50}$, comparado aos das demais variáveis, indicam ser esta variável a mais adequada para determinação
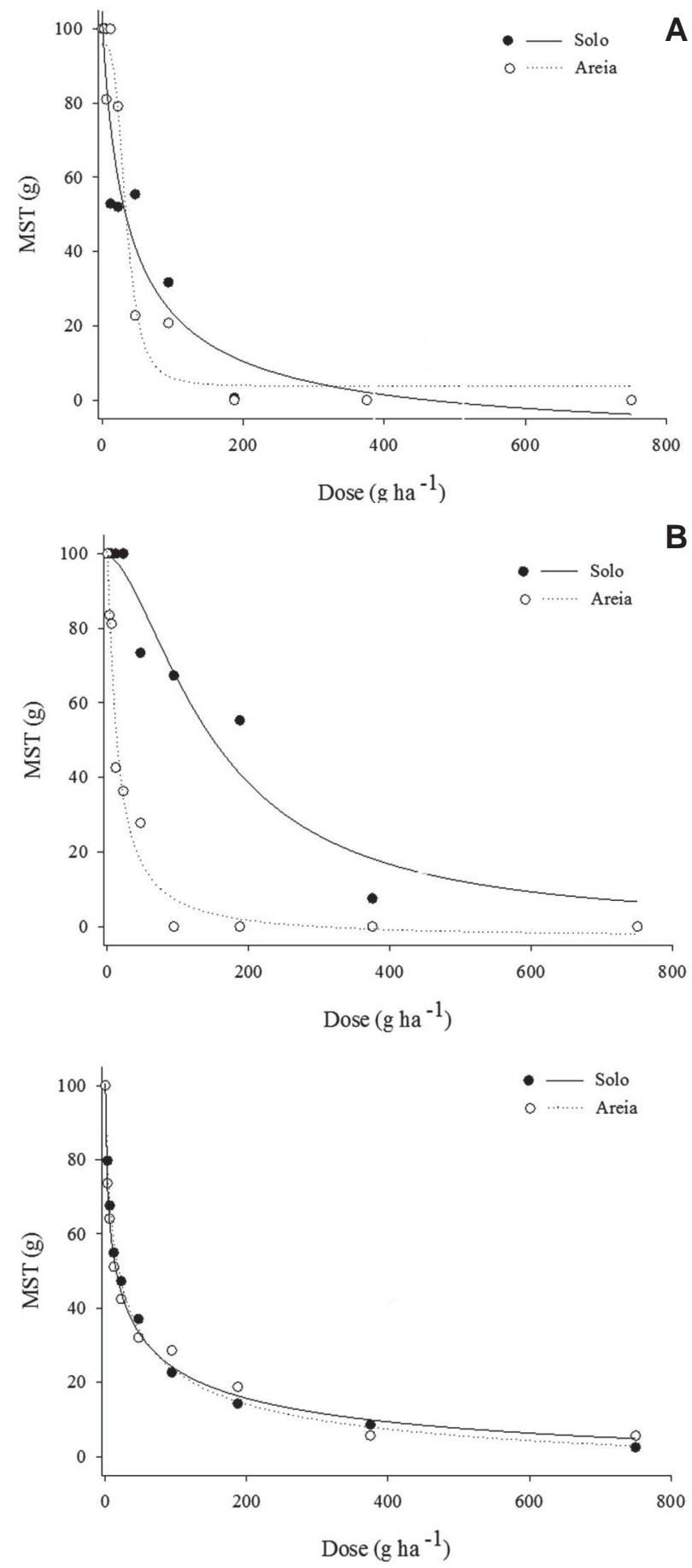

Figura 2. Curva de dose-resposta para matéria seca total de plantas de beterraba (a), tomate (b) e pepino (c) em solo e em areia, em função de doses crescentes do herbicida 2,4-D. 
de baixos níveis de resíduos de 2,4-D no solo, sendo que a beterraba (Figura 3a) foi a espécie que apresentou maior sensibilidade, sendo observados valores de $I_{50}$ de 8,44 e de 7,50 $\mathrm{g} \mathrm{ha}^{-1}$, para solo e areia, respectivamente (Tabela 1).

Embora a persistência no solo do 2,4-D seja considerada de curta a média e o período residual não exceda a quatro semanas em solos argilosos e clima quente (Silva et al., 2007b), esse herbicida pode -se tornar problema quando utilizado em mistura com o glyphosate, para
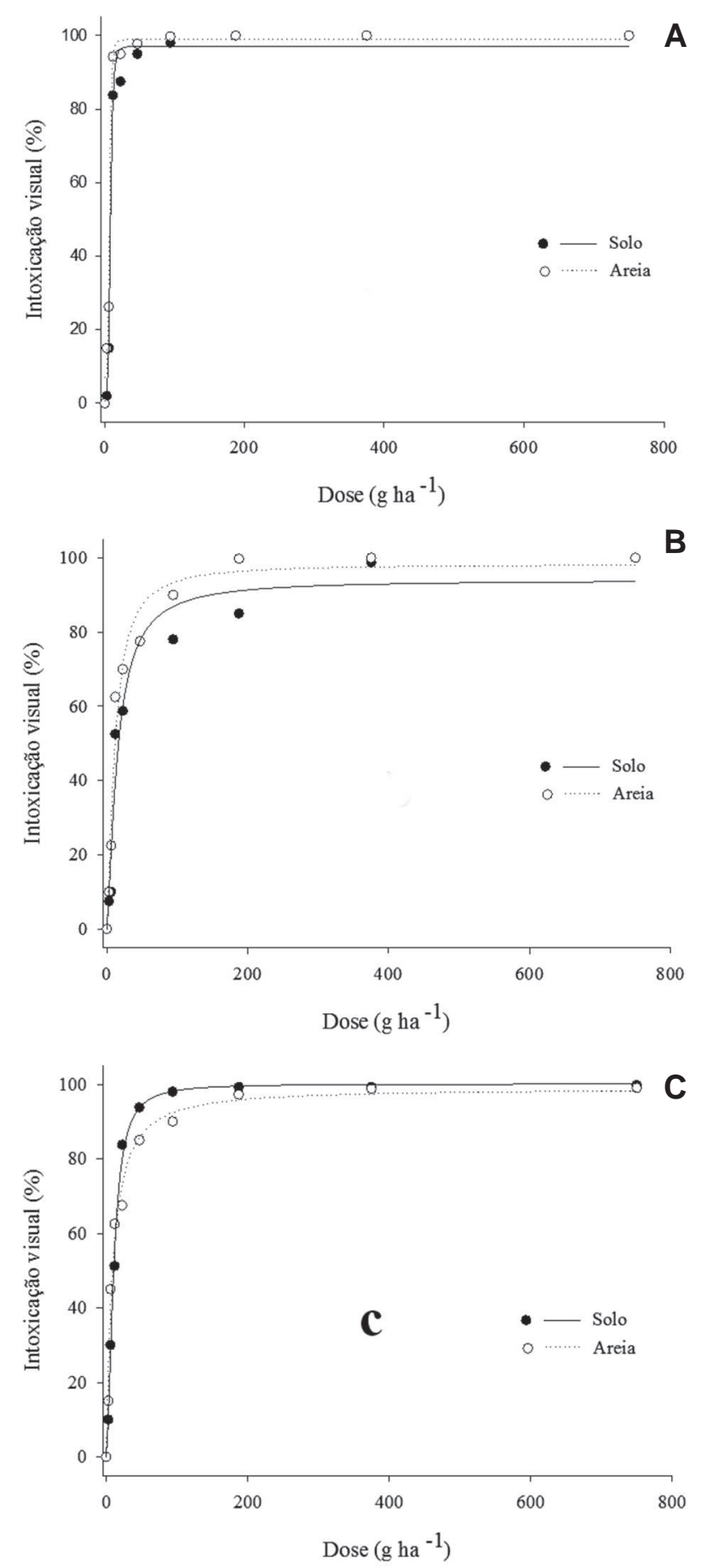

Figura 3. Curva de dose-resposta para intoxicação visual de plantas de beterraba (a), tomate (b) e pepino (c) em solo e em areia, em função de doses crescentes do herbicida 2,4-D. dessecação de áreas sob plantio direto, pois pode causar intoxicação em espécies sensíveis. O uso de plantas indicadoras de baixas concentrações do 2,4-D, como a beterraba, pode ser uma alternativa para avaliar o momento ideal para o cultivo, visto que o uso de sintomas visuais de intoxicação mostrou-se viável mesmo para pequenas doses do herbicida.

Em relação ao picloram, observou-se que a altura de planta (ALT) da beterraba (Figura 5a) e tomate (Figura 5b) reduziu com o aumento da dose do herbicida. $\mathrm{O}$ tomate mostrou-se a espécie com maior sensibilidade, em relação às demais espécies. A utilização das doses 185,22 e 144,33 $\mathrm{g} \mathrm{ha}^{-1}$ de picloram, em solo e areia, respectivamente, foram suficientes para reduzir em 50\% a altura das plantas. Para o pepino (Figura 5c), não foi possível estimar o $I_{50}$ para o herbicida picloram, por meio do modelo proposto por Seefeldt et al. (1995) (Tabela 2).

Em bioensaios para avaliar a presença de resíduos de picloram em solos submetidos à fitorremediação com Eleusine coracana, já foi relatada a redução significativa do porte das plantas de tomate, quando cultivadas em solo que recebeu o herbicida e não foi efetuada a fitorremediação; essa redução também foi proporcional ao nível do picloram no solo (Carmo et al., 2008). No entanto, em trabalho de D'Antonino et al. (2009), o pepino foi utilizado como bioindicadora do picloram em estudos de lixiviação. Possivelmente, a ALT não seja a variável mais indicada para avaliação de resíduos de picloram, visto que os mesmos autores indicaram altos valores de intoxicação visual no pepino, sendo essa a variável mais indicada quando se deseja utilizar o pepino como bioindicadora.

Quanto à MST, observa-se que o tomate foi a espécie mais sensível à presença de picloram, apresentando $\mathrm{I}_{50}$ de 127,26 e 80,00 $\mathrm{g} \mathrm{ha}^{-1}$, respectivamente, para solo e areia (Figura 6b). Para a mesma variável, o pepino (Figura 6c)

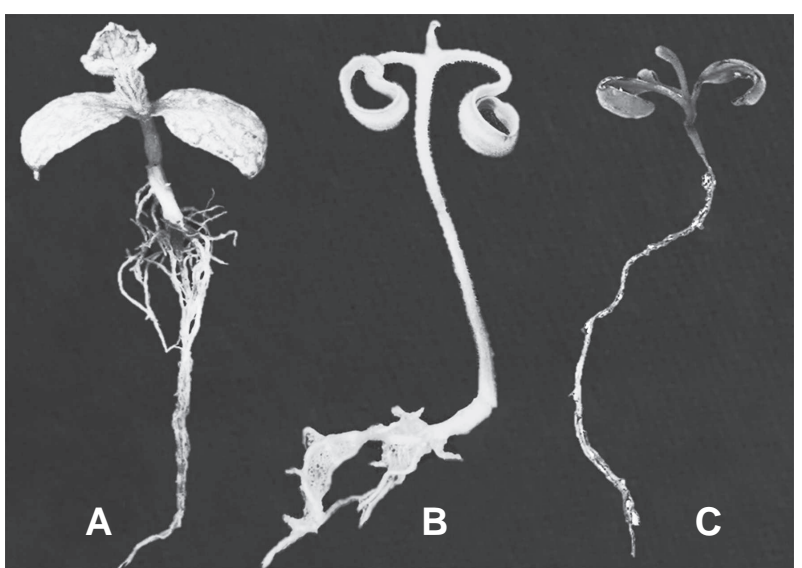

Figura 4. Sintomas de intoxicação visual de plantas de pepino (a), tomate (b) e beterraba (c) com a aplicação dos herbicidas auxínicos. 
demonstrou maior tolerância aos resíduos do picloram, sendo necessárias as doses de 656,81 e 76,51 $\mathrm{g} \mathrm{ha}^{-1}$ para redução de $50 \%$ da MST, respectivamente, em solo e areia (Tabela 2).

O potencial fitotóxico foi crescente para todas as espécies, ou seja, com o aumento da dose observou-se a evolução dos sintomas visuais de intoxicação (Figura 4), com mortalidade acentuada nas maiores doses, principalmente em substrato areia (Figuras 7). Para INT, o tomate


Figura 5. Curva de dose-resposta da altura de plantas de beterraba (a), tomate (b) e pepino (c) em solo e em areia, em função de doses crescentes do herbicida picloram.
(Figura 7b) foi a espécie mais sensível aos resíduos de picloram, apresentando valores do $I_{50}$ bem inferiores aos das demais espécies, os quais foram respectivamente 14,17 e 13,90 $\mathrm{g} \mathrm{ha}^{-1}$, respectivamente, para solo e areia (Tabela 2). Para esta variável, assim como para os resíduos de 2,4$\mathrm{D}$, foram observados menores valores de $I_{50}$ em resposta à aplicação de picloram, o que indica que a variável é adequada para determinação de baixos níveis de resíduos desses herbicidas no solo.
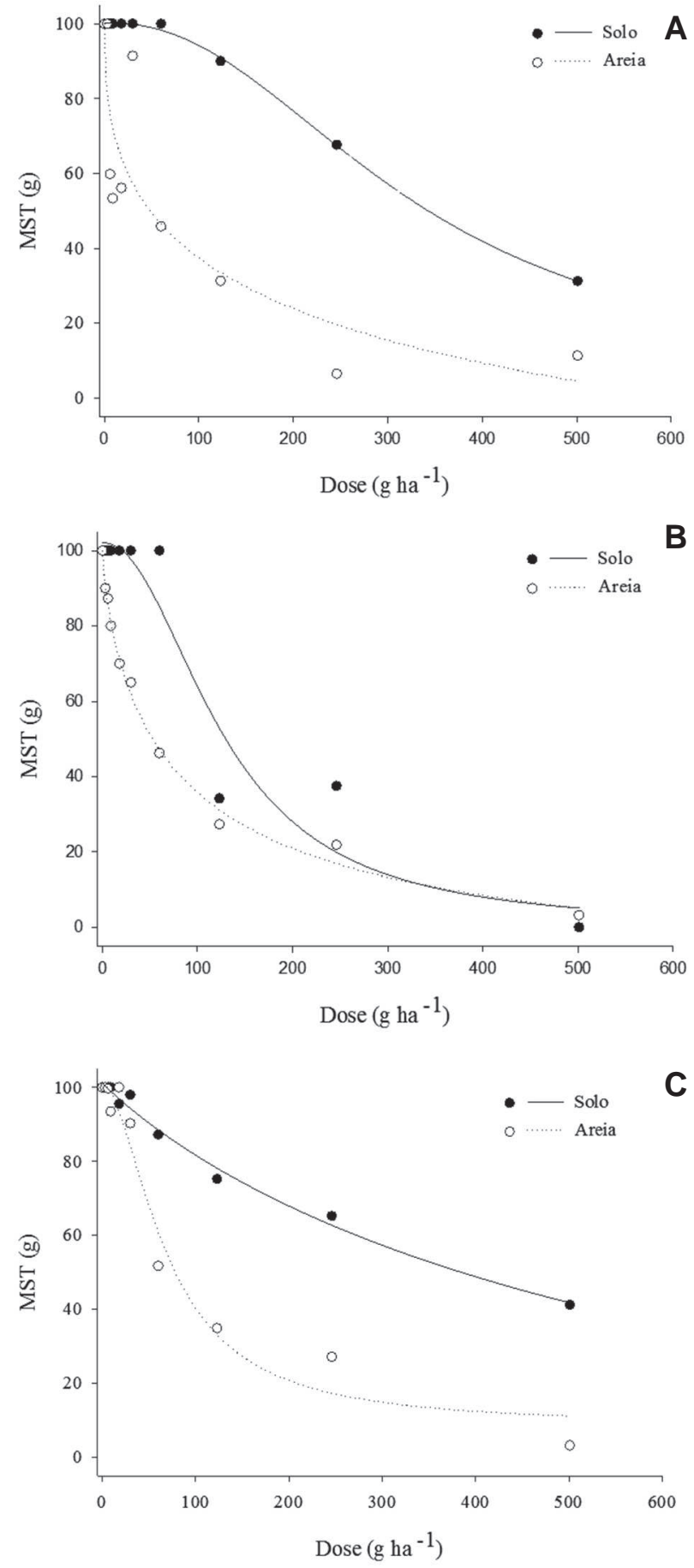

Figura 6. Curva de dose-resposta da matéria seca total de beterraba (a), tomate (b) e pepino (c) em solo e em areia, em função de doses crescentes do herbicida picloram.

Rev. Ceres, Viçosa, v. 60, n.3, p. 354-362, mai/jun, 2013 
Tabela 1. Coeficientes do modelo de regressão $\mathrm{w}=y 0+a / 1+\left(\mathrm{x} / I_{50}\right)^{\mathrm{b}}$ para as variáveis: altura de plantas (ALT), matéria seca total (MST) e intoxicação visual (INT) em resposta a aplicação de 2,4-D, onde $a$ representa a diferença entre os limites superior e inferior da curva, $b$ a declividade da curva e $I_{50}$ é a dose que proporciona $50 \%$ do valor da variável dependente

\begin{tabular}{|c|c|c|c|c|c|c|}
\hline $\begin{array}{l}\text { Variáveis } \\
\text { avaliadas }\end{array}$ & Bioindicadora & Substrato & $a^{*}$ & $b^{*}$ & $I_{50}$ & $\mathbf{R}^{2}$ \\
\hline \multirow{6}{*}{ ALT } & \multirow[t]{2}{*}{ Beterraba } & Latossolo Vermelho-Amarelo & 119,04 & $-1,56$ & 102,58 & 0,99 \\
\hline & & Areia & 107,68 & $-0,54$ & 3,55 & 0,99 \\
\hline & \multirow[t]{2}{*}{ Pepino } & Latossolo Vermelho-Amarelo & 148,63 & $-0,34$ & 193,38 & 0,98 \\
\hline & & Areia & 106,63 & $-0,73$ & 19,76 & 0,99 \\
\hline & \multirow[t]{2}{*}{ Tomate } & Latossolo Vermelho-Amarelo & 100,76 & $-2,81$ & 243,25 & 0,99 \\
\hline & & Areia & 93,90 & $-1,39$ & 30,07 & 0,98 \\
\hline \multirow{6}{*}{ MST } & \multirow[t]{2}{*}{ Beterraba } & Latossolo Vermelho-Amarelo & 116,49 & $-0,87$ & 38,46 & 0,96 \\
\hline & & Areia & 99,71 & $-3,48$ & 33,99 & 0,98 \\
\hline & \multirow[t]{2}{*}{ Pepino } & Latossolo Vermelho-Amarelo & 107,87 & $-0,64$ & 23,25 & 0,99 \\
\hline & & Areia & 108,55 & $-0,55$ & 19,88 & 0,99 \\
\hline & \multirow[t]{2}{*}{ Tomate } & Latossolo Vermelho-Amarelo & 99,48 & $-1,64$ & 151,02 & 0,98 \\
\hline & & Areia & 103,42 & $-1,15$ & 14,05 & 0,99 \\
\hline \multirow{6}{*}{ INT } & \multirow[t]{2}{*}{ Beterraba } & Latossolo Vermelho-Amarelo & 97,55 & 4,96 & 8,44 & 0,99 \\
\hline & & Areia & 106,00 & 5,87 & 7,50 & 0,99 \\
\hline & \multirow[t]{2}{*}{ Pepino } & Latossolo Vermelho-Amarelo & 100,09 & 1,78 & 10,44 & 0,99 \\
\hline & & Areia & 98,88 & 1,12 & 8,84 & 0,99 \\
\hline & \multirow[t]{2}{*}{ Tomate } & Latossolo Vermelho-Amarelo & 94,02 & 1,36 & 14,97 & 0,98 \\
\hline & & Areia & 98,31 & 1,39 & 11,36 & 0,99 \\
\hline
\end{tabular}

Tabela 2. Coeficientes do modelo de regressão $\mathrm{w}=y 0+a / 1+\left(\mathrm{x} / I_{50}\right)^{\mathrm{b}}$ para as variáveis: altura de plantas (ALT), matéria seca total (MST) e intoxicação visual (INT) em resposta a aplicação de picloram, onde $a$ representa a diferença entre os limites superior e inferior da curva, $b$ a declividade da curva e $I_{50}$ é a dose que proporciona $50 \%$ do valor da variável dependente

\begin{tabular}{|c|c|c|c|c|c|c|}
\hline $\begin{array}{l}\text { Variáveis } \\
\text { avaliadas }\end{array}$ & Bioindicadora & Substrato & $a^{*}$ & $b^{*}$ & $I_{50}$ & $\mathbf{R}^{2}$ \\
\hline \multirow{6}{*}{ ALT } & \multirow[t]{2}{*}{ Beterraba } & Latossolo Vermelho-Amarelo & 55,59 & $-3,38$ & 198,17 & 0,99 \\
\hline & & Areia & 64,84 & $-4,58$ & 146,57 & 0,99 \\
\hline & \multirow[t]{2}{*}{ Pepino } & Latossolo Vermelho-Amarelo & - & - & - & - \\
\hline & & Areia & 100,36 & $-2,44$ & 200,73 & 0,99 \\
\hline & \multirow[t]{2}{*}{ Tomate } & Latossolo Vermelho-Amarelo & 100,29 & $-3,19$ & 185,22 & 0,99 \\
\hline & & Areia & 118,76 & $-0,94$ & 144,33 & 0,99 \\
\hline \multirow{6}{*}{ MST } & \multirow[t]{2}{*}{ Beterraba } & Latossolo Vermelho-Amarelo & 94,98 & $-2,27$ & 325,56 & 0,99 \\
\hline & & Areia & 119,74 & $-0,55$ & 39,30 & 0,95 \\
\hline & \multirow[t]{2}{*}{ Pepino } & Latossolo Vermelho-Amarelo & 136,25 & $-0,95$ & 656,81 & 0,99 \\
\hline & & Areia & 98,16 & $-1,45$ & 76,51 & 0,99 \\
\hline & \multirow[t]{2}{*}{ Tomate } & Latossolo Vermelho-Amarelo & 102,04 & $-2,14$ & 127,26 & 0,96 \\
\hline & & Areia & 118,27 & $-0,76$ & 80,00 & 0,99 \\
\hline \multirow{6}{*}{ INT } & \multirow[t]{2}{*}{ Beterraba } & Latossolo Vermelho-Amarelo & 206,16 & 0,54 & 574,55 & 0,98 \\
\hline & & Areia & 106,44 & 1,08 & 41,03 & 0,99 \\
\hline & \multirow[t]{2}{*}{ Pepino } & Latossolo Vermelho-Amarelo & 137,65 & 1,40 & 343,16 & 0,98 \\
\hline & & Areia & 114,39 & 0,87 & 41,95 & 0,98 \\
\hline & \multirow[t]{2}{*}{ Tomate } & Latossolo Vermelho-Amarelo & 94,19 & 1,73 & 14,17 & 0,98 \\
\hline & & Areia & 98,22 & 1,63 & 13,90 & 0,99 \\
\hline
\end{tabular}

Tabela 3. Razão de Adsorção (RA) dos herbicidas 2,4-D e picloram para diferentes plantas bioindicadoras

\begin{tabular}{|c|c|c|c|c|c|c|}
\hline \multirow{2}{*}{$\begin{array}{l}\text { Herbicida } \\
\text { Cultura }\end{array}$} & \multicolumn{3}{|c|}{ 2,4-D } & \multicolumn{3}{|c|}{ Picloram } \\
\hline & I50 solo & I50 areia & $\mathbf{R A}$ & I50 solo & $I 50$ areia & $\mathbf{R A}$ \\
\hline Beterraba & 8,44 & 7,5 & 0,13 & 574,55 & 41,03 & 13,00 \\
\hline Pepino & 10,44 & 8,84 & 0,18 & 343,16 & 41,95 & 7,18 \\
\hline Tomate & 14,97 & 11,36 & 0,32 & 14,17 & 13,9 & 0,019 \\
\hline
\end{tabular}

Rev. Ceres, Viçosa, v. 60, n.3, p. 354-362, mai/jun, 2013 
Já foi relatado que a contaminação de solos com 2,4-D + picloram interfere no crescimento de plantas de tomate e de pepino, havendo redução na altura de plantas a partir de doses de 0,04 $\mathrm{L} \mathrm{ha}^{-1}$. Na medida em que as doses foram aumentadas, essa diferença tornou-se cada vez maior, o que demonstra a alta sensibilidade dessas espécies (pepino e tomate) aos herbicidas auxínicos, levando a mudanças no seu metabolismo (Nascimento \& Yamashita, 2009).
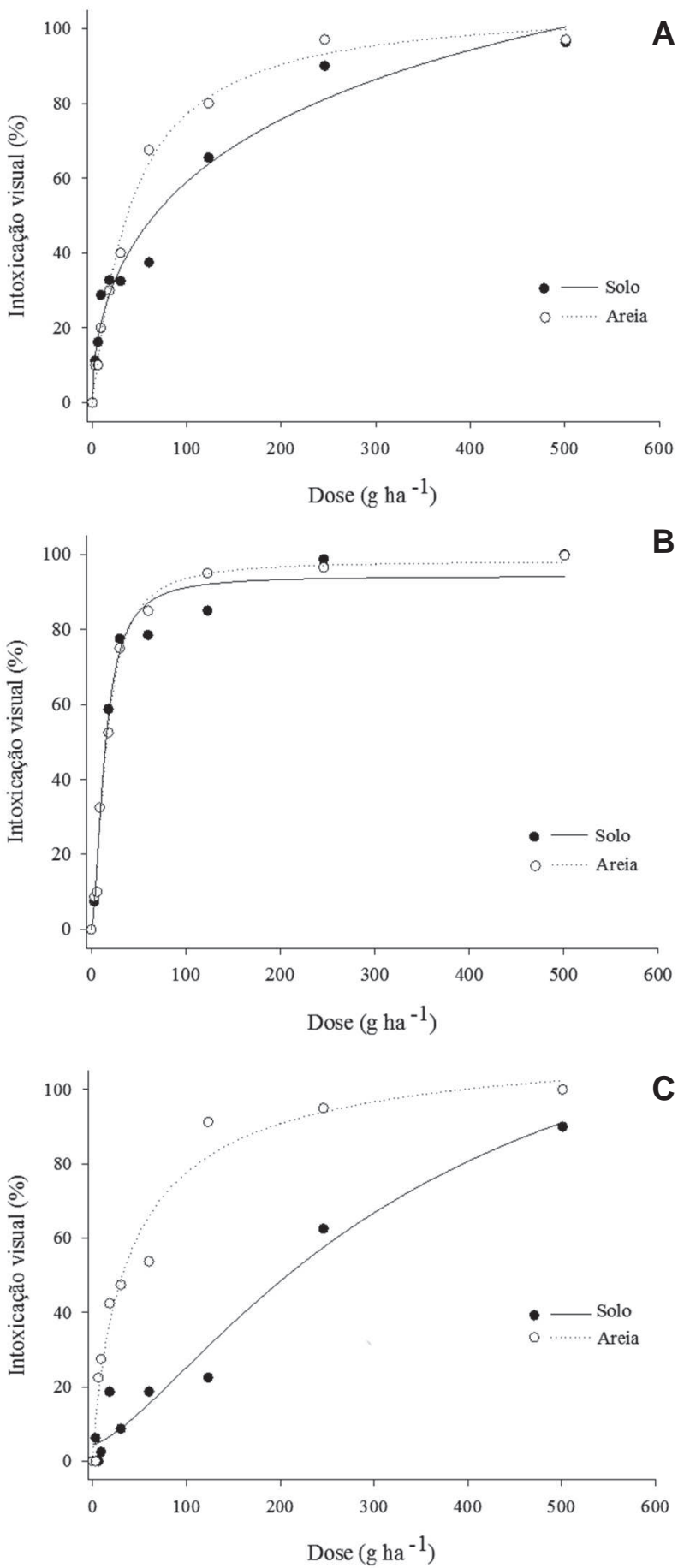

Figura 7. Curva de dose-resposta da intoxicação visual de beterraba (a), tomate (b) e pepino (c) em solo e em areia, em função de doses crescentes do herbicida picloram.
De acordo com a Tabela 3, tem-se que as diferentes espécies apresentaram diferentes valores de razão de adsorção (RA) e, consequentemente, diferentes capacidades de sorção para um mesmo herbicida. O valor de RA para o herbicida 2,4-D foi menor para a beterraba, enquanto, para o picloram, o tomate mostrou-se mais sensível, apresentando valor inferior aos das demais bioindicadoras. Esses resultados estão de acordo com os observados para as demais variáveis, confirmando a sensibilidade diferencial de cada planta aos herbicidas estudados.

\section{CONCLUSÕES}

A beterraba e o tomate foram as espécies que apresentaram maior sensibilidade ao 2,4-D e picloram, respectivamente, sendo que os sintomas visuais de intoxicação mostraram-se as variáveis mais indicadas para a avaliação da presença do resíduo dos herbicidas.

\section{AGRADECIMENTOS}

Os autores agradecem ao Conselho Nacional de Desenvolvimento Científico e Tecnológico (CNPq), à Coordenação de Aperfeiçoamento de Pessoal de Nível Superior (CAPES) e à Fundação de Amparo a Pesquisa do Estado de Minas Gerais (FAPEMIG), pelo apoio financeiro na execução deste trabalho.

\section{REFERÊNCIAS}

ALAM (Asóciacion Latinoamericana de Malezas) (1974) Recomendaciones sobre unificación de los sistemas de avaluacion en ensayos de control de malezas. Bogotá, ALAM, $1: 35-38$.

Belo AF, Santos EA, Santos JB, Ferreira LR, Silva AA, Cecon PR \& Silva LL (2007) Fitorremediação de solo adubado com composto orgânico e contaminado com trifloxysulfuron-sodium. Planta Daninha, 25:251-258.

Brasil (2013) Ministério da Agricultura, Pecuária e Abastecimento. Coordenação-Geral de Agrotóxicos e Afins. Disponível em: <.http://extranet.agricultura.gov.br/agrofit__cons/ principal_agrofit_cons>. Acessado em: 8 de abril de 2013.

Carmo ML, Procopio SO, Pires FR, Cargnelutti Filho A, Braz GBP, Silva WFP, Barroso ALL, Silva GP, Carmo EL, Braz AJBP, Pacheco LP (2008) Influência do período de cultivo do capimpé-de-galinha-gigante (Eleusine coracana) na fitorremediação de solo contaminado com picloram. Planta Daninha, 26:601609.

D'antonino L. Silva AA, Ferreira LR, Cecon PR, Quirino ALS, Freitas LHL (2009) Efeitos de culturas na persistência de herbicidas auxí-nicos no solo. Planta daninha, 27:371-378.

Filizola HF, Ferracini VL, Sans LMA, Gomes MAF \& Ferreira CJA (2002) Monitoramento e avaliação do risco de contaminação por pesticidas em água superficial e subterrânea na região de Guairá. Pesquisa Agropecuária Brasileira, 37:659-667.

Nascimento ER \& Yamashita OM (2009) Desenvolvimento inicial de olerícolas cultivadas em solos contaminados com resíduos de 2,4-d + picloram. Semina. Ciências Agrárias, 30:47-54. 
Monquero PA, Amaral LR, Binha DP, Silva AC \& Silva PV (2008) Potencial de lixiviação de herbicidas no solo submetidos a diferentes simulações de precipitação. Planta Daninha, 26:403408 .

Nyffeler A, Gerber HR, Hurle K, Pestemer W \& Schmidt RR (1982) Collaborative studies of dose-response curves obtained with different bioassay methods for soil-applied herbicides. Weed Research., 22:213-222.

Petacci F, Momesso MA, Latrônico AH, Neves MG \& Freitas SS (2001) Potencial fitotóxico de frutos de Stryphnodendron polyphyllum. Revista Ecossistema, 26:187-190.

Procópio SO, Carmo ML, Pires FR, Cargnelutti Filho A, Braz GBP, Silva WFP, Barroso ALL, Silva GP, Carmo EL \& Braz AJBP (2008) Fitorremediação de solo contaminado com picloram por capim-pé-de-galinha-gigante (Eleusine coracana). Revista Brasileira de Ciência Solo, 32:2517-2524.

Salvador JO, Moreira A \& Muraoka T (1999) Efeito da omissão combinada de $\mathrm{N}, \mathrm{P}, \mathrm{K}$ e S nos teores foliares de macronutrientes em mudas de goiabeira. Scientia Agricola, 56:501-507.

Santos JB, Silva AA, Ferreira LR, Procópio SO \& Pires FR (2007) Fitorremediação de áreas contaminadas por herbicidas. In: Silva AA \& Silva JF (Eds.) Tópicos em manejo de plantas daninhas. Viçosa, Editora UFV. p. 210-329.
Seefeldt SS, Jensen SE \& Fuerst EP (1995) Log-logistic analysis of herbicide dose-response relationship. Weed Technology, 9:218227.

Silva AA, Vivian R \& Oliveira Júnior RS (2007a) Herbicidas: Comportamento no solo. In: Silva AA \& Silva JF (Eds). Tópicos em manejo de plantas daninhas. Viçosa, Editora UFV. p. 156-209.

Silva AA, Ferreira FA \& Ferreira LR (2007b) Herbicidas: classificação e mecanismo de ação. In: Silva AA \& Silva JF (Eds). Tópicos em manejo de plantas daninhas. Viçosa, Editora UFV. p. 83-148.

Souza AP, Prates HT, Ferreira FA, Reis EL \& Jordão CP (1999) Lixiviação do glyphosate e do imazapyr em solos com diferentes texturas e composição química. II. Método analítico. Planta Daninha, 17:245-262.

Souza AP, Loures EG, Silva JF \& Ruiz HA (1996) Efeito do oxyfluorfen, 2,4-D e glyphosate na atividade microbiana de solos com diferentes texturas e conteúdo de matéria orgânica. Planta Daninha, 14:55-64.

Thill D (2003) Growth regulator herbicides. In: Weller SC, Thill D, Bridges DC, Van Scoyoc GE, Graveel JG, Turco Júnior Rf, Goldsbrough P, Ruhl GE, Holt HA, Reicher ZJ \& Whitford F (Eds.) Herbicide action course. West Lafayette, Purdue University. p. 267-291. 